\title{
ADEQUABILIDADE DOS PRINCIPAIS MODELOS DE PERIODIZAÇÃO DO TREINAMENTO ESPORTIVO
}

\author{
DR. ESTÉLIO HENRIQUE MARTIN DANTAS \\ Laboratório de Biociências da Motricidade Humana (LABIMH) da Universidade Federal \\ do Estado do Rio de Janeiro (UNIRIO) (Rio de Janeiro - Rio de Janeiro - Brasil) \\ Doutorado em Educação Física pela Universidade do Estado do Rio de Janeiro (UERJ) \\ (Rio de Janeiro - Rio de Janeiro - Brasil) \\ e-mail: estelio.dantas@unirio.br
}

\section{MS. ERIK SALUM DE GODOY}

Universidade Veiga de Almeida (UVA) (Rio de Janeiro - Rio de Janeiro - Brasil) Mestrado em Ciência da Motricidade Humana pela Universidade Castelo Branco (UCB)

(Rio de Janeiro - Rio de Janeiro - Brasil)

e-mail: eriksalum6I@gmail.com

\section{MS. CARLOS ALBERTO SPOSITO-ARAUJO}

Laboratório de Biociências da Motricidade Humana (LABIMH) da Universidade Federal do

Estado do Rio de Janeiro (UNIRIO) (Rio de Janeiro - Rio de Janeiro - Brasil)

Mestrado em Engenharia Biomédica pelo Instituto Alberto Luiz Coimbra de Pós-graduação e

Pesquisa de Engenharia (COPPE) da Universidade Federal do Rio de Janeiro (UFRJ)

(Rio de Janeiro - Rio de Janeiro - Brasil)

e-mail: carlos@sposito.com.br

\section{MS. ARTUR LUÍS BESSA DE OLIVEIRA}

Instituto de Genética e Bioquímica da Universidade Federal de Uberlândia (UFU)

(Uberlândia - Minas Gerais - Brasil)

Mestrado em Ciência da Motricidade Humana pela Universidade Castelo Branco (UCB)

(Rio de Janeiro - Rio de Janeiro - Brasil)

e-mail: arturbessa@globo.com

\section{MS. RAFAEL CORDEIRO AZEVEDO IN MEMORIAM}

Mestrado em Ciência da Motricidade Humana pela Universidade Castelo Branco (UCB)

\section{DR. MANOEL JOSÉ GOMES TUBINO IN MEMORIAM}

Doutorado em Educação Física pela Universite Libre de Bruxelles (Bélgica) 


\author{
DR. ANTÔNIO CARLOS GOMES \\ Universidade Federal de São Paulo (UNIFESP) Doutorado em Teoria e \\ Metodologia da Educação Física e Treinamento Desportivo pela \\ Academia Nacional de Cultura Física da Rússia (Moscou - Rússia) \\ São Paulo - São Paulo - Brasil) \\ e-mail:contatacgomes@gmail.com
}

\section{RESUMO}

O estudo identificou, sob o prisma da adequabilidade, qual o melhor modelo de periodização do treinamento esportivo (PTE). Foram considerados dois indicadores: a estrutura da periodização e a forma de modulação da carga. Foi utilizada a metanálise como ferramenta metodológica. 103 citações foram levantadas numa pesquisa sistemática, em bases de dados. A avaliação pelos critérios estabelecidos indicou cinco modelos de PTE passíveis de estudo. A análise estatística, empregando o tamanho efeito (TE), gerou um Índice de Adequabilidade (IADEQ) e a classificação dos modelos em: Muito Bom - Matveev (IADEQ = 3,44; TE = 1, I3); Bom - Verkhoshansky (IADEQ = 2,88; TE =0,57), Bompa (IADEQ = 2,66; TE =0,34) e Regular - ATR (IADEQ = 1,96; TE =-0,37), Forteza (IADEQ = 2,25; TE =-0,07).

PALAVRAS-CHAVE: Educação física e treinamento; metanálise; desempenho atlético; atividade motora.

\section{INTRODUÇÃO}

Periodização é o planejamento geral e detalhado do tempo disponível para o treinamento, de acordo com os objetivos intermediários e perfeitamente estabelecidos, respeitando-se os princípios científicos do treinamento desportivo (DANTAS, 2003; TUBINO; MOREIRA, 2003). A ideia de periodizar o treinamento não é nova. Seus fundamentos remontam à Grécia Antiga, aplicados na preparação militar, e, na atualidade, esse conceito, desenvolvido ao longo do tempo, tem por objetivo melhorar o desempenho esportivo.

Barbosa et al. (2004), em pesquisa preliminar, visando fundamentar a elaboração de critérios de identificação e avaliação dos modelos de periodização, levantaram os seguintes aspectos a serem considerados: ser específica quanto à estrutura; estar direcionada a um tipo de atividade; atender ao calendário; distribuir as cargas; possuir formato de macro, meso e microciclos; atender as exigências do desporto; ser condizente no nível de excelência do atleta; contemplar os objetivos; e adequar-se ao momento do plano de expectativa. Partindo deste pressuposto, Azevedo (2005) aprofundou os estudos e definiu os critérios de classificação dos modelos de periodização, agrupando-os em: adequabilidade; abrangência; e aplicabilidade. 
Reconhecer qual a forma de periodização mais adequada para cada uso - quer nas diversas situações de treinamento esportivo, quer nos programas de condicionamento físico para a saúde - é uma dúvida que assola os treinadores constantemente (MILISTETD et al., 2008; RAMALHO; MARTINS, 2003).

Um dos aspectos sob os quais os distintos modelos de periodização existentes podem ser analisados é por meio de sua adequabilidade, que, segundo Barbosa et al. (2004), é passível de ser avaliada por meio de dois indicadores: a estrutura da periodização; e a organização da variação das cargas.

Zakharov e Gomes (1992, p. 232) afirmam, sobre a estrutura da periodização, que "sem estar apoiado numa ideia clara de estrutura de preparação elaborada, não é possível dirigir efetivamente o processo de treinamento". Portanto, para que se pudesse avaliar a estrutura da periodização, esta foi categorizada em três níveis: completa; adaptada; e indefinida.

Foi classificado como completa, segundo Azevedo (2005), o modelo de periodização que possuir três períodos: Preparação; Competição; e Transição. Por sua vez, classificar-se-á como adaptada, como o próprio nome sugere, qualquer uma que possuir apenas um ou dois dos períodos citados. Já a categoria indefinida aplica-se nos casos de não ser identificada a forma de estruturação da periodização observada. Alguns autores se limitam a explicar algum ponto da periodização e não expõem sua estrutura com clareza.

O segundo indicador de avaliação da adequabilidade de um modelo de periodização refere-se à modulação das cargas. As referências bibliográficas disponíveis apresentam quase dez diferentes elementos de carga, tais como: volume; intensidade; densidade; duração; frequência; natureza dos exercícios; duração e natureza dos intervalos de repouso; número de repetições; e magnitude do estímulo (BOMPA, 2002; FORTEZA; RIBAS, 1988; MANSO et al., 2002; SOUZA; ZUCAS, 2003). No entanto, é notável a importância de dois desses elementos - o volume e a intensidade -, fato que é confirmado por Verkhoshansky (1990), ao expor que os índices gerais mais utilizados de carga de treinamento são o volume e a intensidade, e por Dantas (2003), quando afirma que um dos princípios científicos fundamentais do treinamento esportivo é a interdependência entre o volume e a intensidade.

Vale sempre ressaltar que, de acordo com Forteza e Ribas (1988), o aumento do volume e da intensidade de treinamento provoca desvios no estado funcional dos diferentes sistemas e órgãos, além do aparecimento e da intensificação dos processos de fadiga.

Os critérios observados para avaliação da modulação das cargas são três: variação de predominância; predominância de volume; ou predominância de intensidade. A variação de predominância ocorre quando o autor prevê as cargas de 
acordo com o princípio científico de interdependência entre volume e intensidade, ou seja, as cargas começam de uma forma e se invertem durante o macrociclo. Por sua vez, a predominância de volume acontece quando, durante todo o macrociclo, as cargas de volume são superiores à de intensidade. Por fim, a predominância de intensidade apresenta-se quando, durante todo o macrociclo, as cargas de intensidade são superiores à de volume (AZEVEDO, 2005).

Com base nos fundamentos apresentados, o presente estudo tem como objetivo verificar o grau de adequabilidade dos distintos modelos de periodização do treinamento esportivo apresentados nas fontes de consulta avaliadas.

\section{METODOLOGIA}

Para o presente estudo optou-se, como estratégia metodológica, pelo uso da metanálise, que, segundo Thomas e Nelson (2007), é a metodologia utilizada para traduzir os dados nas informações necessárias a esta pesquisa e tem como objetivo combinar e resumir os resultados de vários outros estudos numa síntese matemática, integrando-os com a finalidade de resolver os problemas da revisão tradicional.

Segundo Galvão et al. (2004), a metanálise é indicada: quando os resultados de vários estudos discordam quanto à magnitude ou à direção do efeito; quando ensaios para avaliar um determinado assunto são caros; ou demandam longo tempo para serem realizados. Portanto, a metanálise se adequa perfeitamente ao presente estudo, por ser uma metodologia que permite alcançar os objetivos almejados, já que se trata de um estudo experimental no qual é praticamente impossível se fazer uma intervenção (CASTRO, 200 I; KNAPP, 2002).

A metanálise, segundo Vieira e Hassne (200I), é realizada através de sete etapas: formulação da pergunta; localização e seleção dos estudos; avaliação crítica dos estudos; coleta de dados; análise e apresentação dos dados; interpretação dos dados; e aprimoramento e atualização da revisão.

Em uma busca sistemática pelos termos "(periodização OR periodization OR periodización) AND (treinamento OR training OR entrenamiento)" nas bases de dados full text EMBASE, SPORT DISCUS, MEDLINE e Portal da CAPES, no período de 2004 a 2005, atualizada no segundo semestre de 2008, foram encontradas 12.342 referências. Desse total, foram selecionados os artigos utilizados no estudo.

Como critérios de inclusão foram consideradas quaisquer formas de vinculação de conhecimento (artigo, livro, CD-ROM, hipertexto) que contemplassem o conteúdo selecionado, que contivessem os indicadores de adequabilidade já descritos e que possuíssem conteúdo científico consistente; e, como critério de exclusão, 
as fontes que não permitissem a aplicação integral do método escolhido, por não possuírem os fatores de avaliação de qualidade determinados. Após a aplicação dos critérios de inclusão e exclusão citados, chegou-se a cento e três referências.

Em uma planilha eletrônica as informações das referências obtidas foram tabuladas por critérios, aos quais foram atribuídos escores em função da avaliação da validade, confiabilidade e peso científico de cada estudo, sendo assim atribuído um valor numérico (GALVÃO et al., 2004).

Foram utilizados vinte e um critérios para a determinação da qualidade de cada estudo: ano do término da pesquisa; situação da pesquisa; informação da fonte; se publicada, onde ocorreu a publicação; se publicada em livro, o número de edições; se publicada em periódico, a tiragem; estrutura da periodização; variação das cargas; número de peaks; nível esportivo; aplicabilidade do modelo; nível dos sujeitos testados; qualidade de definição do grupo; classificação de confiança na seleção do desempenho; como esse nível foi determinado; número total de sujeitos no grupo; sexo dos sujeitos; idade média das amostras usadas nos grupos; número médio de anos de experiência com o esporte; status do sujeito; experiência anterior com a tarefa. Por meio deles, foram avaliados a confiabilidade, a validade e o peso científico de cada estudo.

A média aritmética dos escores que cada referência obteve em cada item resulta no escore geral da mesma. Após esse passo calculou-se, por meio de novas médias aritméticas com alcance diferenciado, o índice geral de abrangência do estudo e de um Índice de Adequabilidade (IADEQ) de cada modelo de periodização.

Considerou-se como grupo de controle (GC) o índice geral formado pela reunião dos dados colhidos de todas as fontes; e os grupos experimentais (GE) foram constituídos, respectivamente, pelos índices referentes a cada um dos cinco modelos de periodização do treinamento a serem investigados. A saber: Verkhoshansky (quinze referências); Bompa (quatorze referências); Matveev, ATR e Forteza (treze referências cada um).

A objetividade da tabela foi validada pelo processo de "face validity", no qual se busca o consenso em uma revisão do instrumento. No caso, efetuada por cinco doutores com notório saber na área de treinamento esportivo, conforme procedimento descrito por Thomas e Nelson (2007). A versão inicial passou pela primeira rodada de revisão, que apontou a necessidade de oito modificações, sendo aprovada por unanimidade na segunda revisão.

O método estatístico adequado para o tratamento dos dados de um estudo de metanálise é denominado Tamanho Efeito (TE), que é determinado pela seguinte fórmula (TRIOLA, 2005): 


$$
\begin{array}{cl}
\frac{M_{E}-M_{C}}{S_{C}} & M_{E}=\text { média do grupo experimental } \\
& M_{C}=\text { média do grupo controle } \\
& S_{C}=\text { desvio-padrão do grupo controle }
\end{array}
$$

O instrumento utilizado foi uma tabela desenvolvida no programa de computador Excel 2007.

\section{RESULTADOS E DISCUSSÃO}

Os dados, após coletados e analisados, possibilitaram o estabelecimento da Tabela I, que apresenta os resultados da análise descritiva dos índices estudados. Nela, observa-se que o IADEQ apresenta uma baixa dispersão ( CV < 25\%), sendo, portanto, a média a melhor medida de tendência central (SHIMAKURA, 2008).

TABELA I - Índice de Adequabilidade para a totalidade dos artigos considerados.

\begin{tabular}{|l|l|l|l|l|l|l|l|l|}
\hline Variáveis & $\mathrm{N}$ & $\mathrm{x}$ & $\sigma$ & Md & $\mathrm{s}$ & $\mathrm{a}^{3}$ & $\mathrm{a}^{4}$ & CV\% \\
\hline IADEQ & 103 & 2,33 & 0,09 & 2,44 & 0,98 & $-0,65$ & $-0,42$ & 22,14 \\
\hline
\end{tabular}

$\mathrm{x}=$ média; $\sigma=$ erro padrão da média; $M d=$ mediana; $s=$ desvio-padrão;

$a^{3}=$ assimetria; $a^{4}=$ curtose; $C V \%=$ coeficiente de variação.

Efetuou-se então o cálculo dos quartis referentes à totalidade do conjunto, proporcionando assim a identificação do quartil em que cada um dos modelos de periodização se encontrava. A Tabela 2 apresenta os pontos de corte destes quartis.

TABELA 2 - Percentis característicos dos pontos de corte de cada quartil, do grupo controle.

\begin{tabular}{|c|c|c|l|}
\hline \multirow{1}{*}{$N$} & Quartis & 103 & Conceitos \\
\hline \multirow{4}{*}{ Categorias } & $\mathrm{Q}_{1}$ & $<1,67$ & Insuficiente \\
\cline { 2 - 4 } & $\mathrm{Q}_{25}$ & $1,67-2,43$ & Regular \\
\cline { 2 - 4 } & $\mathrm{Q}_{50}$ & $2,44-3,43$ & Bom \\
\cline { 2 - 4 } & $\mathrm{Q}_{75}$ & 3,44 ou $>$ & Muito Bom \\
\hline
\end{tabular}

Com as informações referentes aos percentis de corte foi possível agrupar os modelos de periodização estudados e, através de suas médias, calcular os seus respectivos $I A D E Q$, classificando-os dentro dos limites dos percentis, como apresentado na Tabela 3. 
TABELA 3 - Resultados do Índice de Adequabilidade (IADEQ) e do conceito, para os diferentes modelos de periodização.

\begin{tabular}{|c|c|c|c|c|c|}
\hline Variáveis & Verkoshanski & Matveev & Bompa & Atr & Forteza \\
\hline IADEQ & 2,88 & 3,44 & 2,66 & 1,96 & 2,25 \\
\hline Conceito & Bom & Muito Bom & Bom & Regular & Regular \\
\hline
\end{tabular}

Os resultados da Tabela 3, de avaliação de cada grupo experimental, demonstraram claramente que, das cento e três referências sobre periodização do treinamento, o modelo de Matveev apresentou uma adequabilidade superior. Isso, em grande parte, se deve à forma com que o modelo Clássico ou Tradicional de Matveev trabalha com a dimensional intensidade, considerando-a como importante componente da carga, fator amplamente reconhecido como determinante do sucesso desportivo, segundo Moreira (2008).

Em seguida, foi calculado o TE, o que possibilitou a categorização dos resultados proposta por Domingues (2008) em: TE pequeno $(<0,2)$; TE moderado $(\cong 0,5) ;$ TE grande $(>0,8)$.

$\mathrm{Na}$ Tabela 4 pode-se observar que os resultados apresentados demonstram que o IADEQ do modelo de Matveev, avaliado segundo o TE, apresentou um valor de I, I3, sendo, dessa forma, classificado como Grande, e significando, portanto, maior o impacto e a relevância do modelo dentro da amostra estudada.

TABELA 4 - Resultados do Tamanho Efeito (TE) de cada modelo de periodização.

\begin{tabular}{|c|c|c|c|c|c|}
\hline Modelos & Verkoshanski & Matveev & Bompa & Atr & Forteza \\
\hline IADEQ & 0,57 & 1,13 & 0,34 & $-0,37$ & $-0,07$ \\
\hline Conceito & Moderado & Grande & Moderado & Pequeno & Pequeno \\
\hline
\end{tabular}

Podemos verificar que o modelo tradicional de Matveev obteve os melhores resultados na Adequabilidade e no TE, o que podemos atribuir ao fato deste modelo ser apropriado, tanto às categorias de base, quanto aos atletas adultos de alto rendimento. Os modelos de Verkhoshansky e de Bompa obtiveram uma relevância intermediária no universo da amostra. Por último, vêm os modelos ATR e Forteza, que obtiveram os piores resultados da amostra, o que talvez esteja diretamente relacionado à escassez de citações sobre os mesmos nos artigos pesquisados para esta metanálise.

Dois aspectos que não puderam ser melhor analisados nesse estudo, em função da carência de dados nas referências consultadas, mas relevantes na avaliação dos modelos de PTE, são o atendimento às exigências do calendário e as caracte- 
rísticas específicas da atividade. Sobre este aspecto incidem as maiores críticas ao modelo clássico de Matveev (GODOY et al. , 2004), cujo desenvolvimento ocorreu no início do período da Guerra Fria, com a situação tensa entre os países de blocos políticoeconômicos antagônicos (socialista x capitalista), em que o esporte também foi palco desta disputa, visando assim obter os melhores resultados em Olimpíadas (DANTAS, 2003; TUBINO; MOREIRA, 2003). Os expressivos resultados obtidos pela extinta União Soviética com o emprego deste modelo ressaltam sua eficácia para situações em que se tem períodos de preparação relativamente longos para períodos competitivos relativamente curtos (GODOY; AZEVEDO, 2004).

Segundo alguns autores, este modelo não atende às situações que exigem a constante participação em competições, mantendo performances esportivas expressivas em condições distintas, em intervalos de tempo de poucas semanas, pois não se viabiliza a progressão de cargas de alto volume e baixa intensidade para cargas de volume relativamente mais baixo e alta intensidade, que proporcionam o peak ao final do ciclo, característica desse modelo (MORENO, 2004; PORTA; SANZ, 2005; ROETERT et al., 2005).

Contemporaneamente, observam-se duas situações comuns no esporte de alto rendimento:

a. quando se necessita obter cerca de nove peaks em uma temporada, como no ATP Tour do tênis profissional; e

b. quando se necessita manter o desempenho em competições prolongadas, que envolvem a participação em uma sequência de eventos ao longo de quatro meses ou mais, por vezes participando simultaneamente de dois a três campeonatos nessa condição, como é caso do futebol profissional.

No primeiro caso, surgem algumas referências recomendando modelos que se caracterizam pela concentração de cargas específicas distribuídas em ciclos de quatorze a vinte oito dias, propostos por Issurin e Kaverin, Verkhoshansky, Navarro (MARTENS; MAES, 2005; PORTA; SANZ, 2005, ROETERT et al., 2005). No segundo caso, propõem-se modelos que enfatizem a resistência, os eventos como estímulos de treino e a distribuição de em ciclos duplos ou triplos (BOMPA, 2002).

Apesar dessa incipiente tendência, observa-se que, mesmo no esporte contemporâneo, quando se trata das categorias de base, há o consenso, até entre os mais severos críticos do modelo clássico, de que este se constitui no 
modelo mais indicado para lograr êxito (MORENO, 2004; UNIERZYSKI, 2005; VERKHOSHANSKY, 1990). Na atualidade, a periodização de Matveev também apresenta amplas possibilidades de sucesso quando a preparação é longa e a prestação esportiva é curta, obtendo êxito na principal competição do macrociclo em 73,3\% dos casos (GARCIA, 2000).

Provavelmente, uma confirmação dessas tendências se efetuaria com uma extensa pesquisa de campo, verificando os modelos de periodização aplicados por comissões técnicas e treinadores de diversas modalidades esportivas, em suas diferentes categorias, as situações em que foram aplicadas e os respectivos resultados. Porém, algumas dificuldades podem ser antecipadas: o conhecimento teórico de treinadores sobre os modelos de periodização para poder identificá-los corretamente; os modelos de organização e administração esportiva que nem sempre proporcionam um calendário regular e, o mais importante, o controle da influência de outros fatores, além da periodização, na determinação do desempenho esportivo.

\section{CONCLUSÃO}

Podemos, assim, determinar que os critérios estabelecidos como nível de adequabilidade responderam a algumas das necessidades de avaliação da periodização. Matveev (1986, 1990) já proclamava a ampla possibilidade de utilização de seu modelo de periodização, corroborada por este estudo.

Ainda são necessários, no entanto, novos estudos que sistematicamente incluam as novas fontes bibliográficas disponíveis e a busca de possibilidades de experimentação prática das premissas levantadas.

\section{The adequability's of main models of periodization of training}

The aim of this study was to identify using the adequability pattern method, which model of periodization suite better to sport training observed at bibliographic research. It utilized as indicators the structure of periodization and the modulation pattern of workload. The methodological tool of this study was meta-analysis. Were appraised 103 citations. Analysis of the collected material, assessed through the statistic tool size-effect (TE made it possible to established an Adequability Index (IADEQ) and then, categorize the models as : Very Good Matveev (IADEQ = 3,44; TE = 1, 13); Good - Verkhoshansky (IADEQ = 2,88; TE = 0,57), Bompa (IADEQ = 2,66; TE =0,34) e Regular - ATR (IADEQ =1,96; TE =-0,37), Forteza $($ IADEQ $=2,25 ;$ TE $=-0,07)$.

KEYWORDS: Physical education and training; meta-analysis; athletic performance; motor activity. 


\section{Adaptabilidad de los principales modelos de periodización del entrenamiento deportivo}

El estudio identificó, bajo prisma de la adaptabilidad, cual es lo mejor modelo de periodización del entrenamiento deportivo (PTE). Fueron considerados dos indicadores: la estructura de la periodización y la forma de modulación de la carga. Fue utilizada la metanálisis como herramienta metodológica. 103 citaciones habían sido levantadas. La evaluación por los criterios establecidos indicó cinco modelos de PTE pasibles de estudio. El análisis estadístico, empleando el tamaño efecto (TE), generó un Índice de Adaptabilidad (IADEQ, y la clasificación de los modelos en: Muy Bueno - Matveev (IADEQ = 3,44; TE = 1, I3); Bueno - Verkhoshansky (IADEQ = 2,88; TE =0,57), Bompa (IADEQ = 2,66; TE =0,34) y Regular - ATR (IADEQ = 1,96; TE = -0,37). Forteza (IADEQ = 2,25; TE = -0,07). PALABRAS CLAVE: Educación y entrenamiento físico; metanálisis; rendimiento atlético; actividad motora.

\section{REFERÊNCIAS}

AZEVEDO, R. C. Adequabilidade, abrangência e aplicabilidade dos modelos de periodização do treinamento esportivo, apurados pela metanálise. 2005. I 4 I f. Dissertação (Mestrado em Ciência da Motricidade Humana). Programa de Pós-graduação Strictu Sensu em Ciência da Motricidade Humana, Universidade Castelo Branco, Rio de Janeiro, 2005.

BARBOSA, L. O. et al. Fundamentos da elaboração de critérios de classificação dos modelos de periodização do treinamento esportivo. In: CONGRESSO INTERNACIONAL DE ATIVIDADE FÍSICA, SAÚDE E ESPORTE - CONAFISE, I ., Rio de Janeiro. Anais... Fitness \& Performance Journal, 2004. v. 3, p. 384-384.

BOMPA, T. O. Periodização: teoria e metodologia do treinamento. São Paulo: Phorte, 2002.

CASTRO, A. A. Revisão sistemática e meta-análise. Compacta: Temas de Cardiologia, v. 3, n. I, p. 5-9, 2001 .

DANTAS, E. H. M. A prática da preparação física. 5. ed. Rio de Janeiro: Shape, 2003.

DOMINGUES, C. A. Estatística aplicada à metodologia da pesquisa científica para temas militares. Rio de Janeiro: EsAO, 2008.

FORTEZA, A.; RIBAS, R. A. Bases metodólogicas del entrenamiento deportivo. La Habana: Científico Técnica, 1988.

GALVÃO, C. M.; SAWADA, N. O.; TREVIZAN, M. A. Revisão sistemática: recurso que proporciona a incorporação das evidências na prática da enfermagem. Revista Latino-Americana de Enfermagem, v. 12, n. 3, p. 549-556, mai./jun. 2004. 
GARCIA, J. M. S. Ejemplo de planificación en nadadores junior de nivel medio. In: CONGRESO DE LA ASOCIACIÓN ESPAÑOLA DE CIENCIAS DEL DEPORTE, I., Barcelona, 2000.

GODOY, E. S; AZEVEDO, R. C. Proposta de adaptação do modelo de periodização ATR para utilização em esportes coletivos e acíclicos. In: I Congresso Internacional de Atividade Física, Saúde e Esporte - CONAFISE, I ., 2004, Rio de Janeiro. Anais... Fitness \& Performance Journal, Rio de Janeiro, v. 3, n. 6, p. 393-393.

GODOY, E. S. et al. Verificação da consistência das críticas à periodização clássica. In: CONGRESSO PAULISTA DE EDUCAÇÃO FÍSICA, 8., 2004, Jundiaí. Anais... Fontoura Editora, Jundiaí, v. I, p. 397-397.

KNAPP, W. P. Intervenções psicossociais em transtornos por uso de psicoestimulantes: uma revisão sistemática. 2002. I 48 f. Dissertação (Mestrado em Medicina). Faculdade de Medicina, Universidade Federal do Rio Grande do Sul, Porto Alegre, 2002.

MANSO, J. M. G.; VALDIVIELSO, M. N.; CABALLERO, J. A. R. Planificación del Entrenamiento. Madrid: Gymnos, 2002.

MARTENS, S. \& MAES, C. Periodization for professional female tennis player. Coaching \& Sport Science Review, v. 36, p. 13-15, aug. 2005.

MATVEEV, L. P. Fundamentos do treino desportivo. Lisboa: Horizonte, 1986.

MATVEEV, L. P. O processo de treino desportivo. 2. ed. Lisboa: Horizonte, 1990.

MILISTETD, M. et al. Concepções dos treinadores acerca do papel da competição na Formação desportiva de jovens jogadores de voleibol. Revista da Educação Física, Maringá, v. 19, n. 2, p. 151-158, abr.jun. 2008.

MOREIRA, A. Testes de campo para monitorar desempenho, fadiga e recuperação em basquetebolistas de alto rendimento. Revista da Educação Física, Maringá, v. 19, n. 2, p. 24I-250, abr./jun. 2008.

MORENO, J. I. M Clarificación de conceptos relacionados con el entrenamiento deportivo. Escuela Abierta, v. 7, p. 55-7I, 2004.

PORTA, J. \& SANZ, D. Periodisation in top level men's tennis. Coaching \& Sport Science Review, v. 36, p. 12-13, aug. 2005.

RAMALHO, V. P. \& MARTINS Jr, J. Influência da periodização do treinamento com pesos na massa corporal magra em jovens adultos do sexo masculino: um estudo de caso. Revista da Educação Física, Maringá, v. 14, n. 2, p. 49-56, jul./dez. 2003.

ROETERT, P.; REID, M.; CRESPO, M. Introduction to Modern Tennis Periodisation. Coaching \& Sport Science Review, 36, p. 2-3, aug. 2005. 
SHIMAKURA, S. E. Coeficiente de Variação. Disponível em: < http://www. est.ufpr.br/ silvia/ CE055/node26.html>. Acesso em: 2 mar. 201 I.

SOUZA, J.; ZUCAS, S. M. Alterações da resistência aeróbia em jovens futebolistas em um período de 15 semanas de treinamento. Revista da Educação Física, Maringá, v. I4, n. I, p. 31-36, jan./jun. 2003.

THOMAS, J. R.; NELSON, J. K. Métodos de pesquisa em atividade física. 5. ed. Porto Alegre: Artmed, 2007.

TRIOLA, M. F. Introdução à estatística. 9. ed. Rio de Janeiro: LTC, 2005.

TUBINO. M. J. G.; MOREIRA, S. B. Metodologia científica do treinamento desportivo. 13. ed. Rio de Janeiro: Shape, 2003.

UNIERZYSKI, P. Periodization for under-I4s. Coaching \& Sport Science Review, v. 36, p. 4-6, Aug. 2005.

VERKHOSHANSKY, I. V. Entrenamiento deportivo: planificación y programación. Barcelona: Martínez Roca, 1990.

VIEIRA, S.; HASSNE, W. S. Metodologia científica para área da saúde. Rio de Janeiro: Elsevier, 2001 .

ZAKHAROV, A.; GOMES, A. C. Ciência do treinamento desportivo. Rio de Janeiro: Palestra Sport, 1992.

Recebido: 16 jul. 2009 Aprovado: I | abr. 20 I I

Endereço de Correspondência: Estélio Henrique Martin Dantas LABIMH/UNIRIO Rua Xavier Sigaud n 290 - sala 40I - Praia Vermelha CEP 22290- 180 Rio de Janeiro / RJ 